\title{
Intermodal Stability of a Coupled-Cavity Semiconductor Laser
}

\author{
ROBERT J. LANG, STUDENT MEMBER, AND AMMON YARIV, FELLOW, IEEE
}

\begin{abstract}
We present an analysis of the steady-state operation of a two-element coupled-cavity laser near a mode hop. The equations of motion for the two cavities and two relevant modes of a longitudinally coupled-cavity laser are reduced to a system of nondimensional nonlinear ordinary differential equations which describe a general two-element laser. The equations are then solved and the stability of their solutions is analyzed. Depending upon the fill factors for the two modes, there exists an intrinsically multimode oscillation for operating conditions under which it was previously thought that no steady state existed. Under conditions where the multimode state is unstable, both of the single-mode states are stable with bistable transitions occurring only on the boundaries of the unstable multimode regimes.
\end{abstract}

\section{INTRODUCTION}

$\mathrm{C}$ OUPLED-CAVITY lasers have recently become the subject of much study because of their potential for single-mode operation under high-speed current operation [1], [2]. In addition, they, like many other two-element lasers [3], have been shown to exhibit bistable behavior. Such behavior makes them suitable for digital optical read/ write operations or candidates for elements of an optical logic system [4]. Although there have been several analyses of longitudinally coupled-cavity lasers (e.g., a $\mathrm{C}^{3}$ laser) at varying levels of approximation [5]-[8], none has treated the problems associated with operation near a mode boundary. Yet, when the current supplied to one of the two cavities is modulated, crossing a mode boundary is almost inevitable [8]. Recently, in a steady-state analysis, Henry and Kazarinov made the claim that there existed regimes of operation near a mode boundary where no steady-state solutions were stable (although they left open the question of what happened in such a regime). In this paper, we analyze the behavior of a two-element coupled-cavity laser near a mode boundary. Although we choose the specific geometry of a longitudinally coupled pair of cavities, the nondimensional equations of motion are equally applicable to any two-element laser (e.g., a laterally coupled cavity [9]). In Section II, we give a brief development of the equations of motion of the laser, using a slowly varying complex frequency approximation [10]. In Section III, we expand around a mode hop in the carrier

Manuscript received July 29, 1985; revised November 7, 1985. This work was supported by Rockwell International and the Office of Naval Research. The work of R. J. Lang was supported by the National Science Foundation.

The authors are with the California Institute of Technology, Pasadena, CA 91125 .

IEEE Log Number 8607703. density plane and develop a set of nondimensional equations which describe the dynamical behavior of the laser. We solve for the dc solutions of the equations and perform a stability analysis upon the solutions. We show that in all regimes of operation, there exists at least one stable solution, and sometimes two; we identify a sufficiency condition for bistable behavior, and we solve for the lines of instability on which a bistable transition occurs. In Section IV, we summarize the important results of the analysis.

\section{Equations of Motion}

We begin by deriving the eigenvalue equation for the modes of a longitudinally coupled-cavity laser illustrated schematically in Fig. 1. It consists of two cavities of length $L_{1}$ and $L_{2}$, separated by a small gap $D$. Although in actual practice one would control the currents $j_{1}$ and $j_{2}$ supplied to each of the two sections, for the purposes of analysis, it is more convenient to treat the carrier densities $n_{1}$ and $n_{2}$ as free parameters, solve for the lasing frequency $\omega$, and then find the currents necessary to support that operating point at a given power level. We choose our time factor as $e^{j \omega t}$. Then a roundtrip self-reproduction condition imposed upon the field in the laser yields the secular equation [5]

$$
\begin{aligned}
& {\left[\frac{\exp \left[-\gamma_{1}\left(n_{1}\right) L_{1}+2 j \omega \mu_{1}\left(n_{1}\right) L_{1} / c\right]}{R_{1}}-1\right]} \\
& \cdot\left[\frac{\exp \left[-\gamma_{2}\left(n_{2}\right) L_{2}+2 j \omega \mu_{2}\left(n_{2}\right) L_{2} / c\right]}{R_{2}}-1\right] \\
& \quad-K_{s}=0
\end{aligned}
$$

where $\gamma_{i}\left(n_{i}\right)$ is the linear gain constant for the $i$ th cavity, $\mu_{i}\left(n_{i}\right)$ is the index of refraction, $R_{i}$ is the reflectivity of the outside mirrors, and $K_{s}$ is a coupling factor which characterizes the gap (taking into account the length and reflectivity of the mirrors bounding the gap) [5]. Equation (1) can be considered [10] an implicit equation for complex $\omega$ as a function of $n_{1}$ and $n_{2}$. For any fixed pair $\left(n_{1}\right.$, $n_{2}$ ), there exists an infinite number of complex $\omega$ solutions; each solution corresponds to a different spatial (longitudinal) mode of the structure. We are not interested in the phase of the optical field, so we can ignore the real part of $\omega$, but the dynamics of the power depend upon the imaginary part. The time evolution of the average photon 

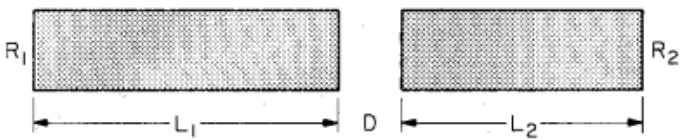

Fig. 1. Schematic of a longitudinally coupled-cavity laser.

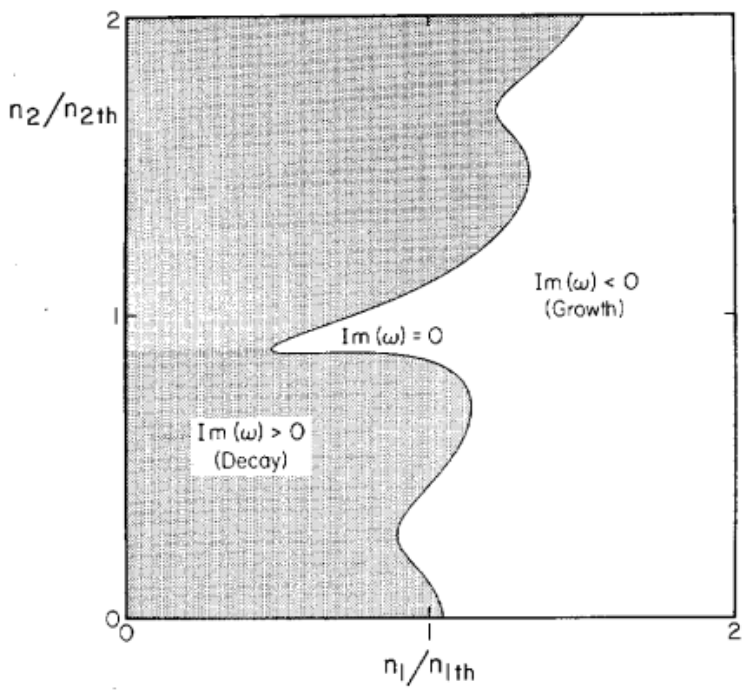

Fig. 2. Plot of the line $\operatorname{Im}(\omega)=0$ for a single mode in the carrier density plane (normalized to threshold values for the uncoupled lasers) for a laser with $L_{1}=200 \mu \mathrm{m}, L_{2}=20 \mu \mathrm{m}, D=1.5$ wavelengths.

density $p_{i}$ in the $i$ th mode obeys

$$
\dot{p}_{i}=-2 \operatorname{Im}\left(\omega_{i}\right) p_{i}+\frac{1}{\tau_{s}} \sum_{j} \beta_{i j} n_{j}
$$

where $n_{j}$ is the carrier density in the $j$ th cavity, $\tau_{s}$ is the spontaneous lifetime, and $\beta_{i j}$ are coefficients representing the fraction of spontaneous emission coupled into the optical field. For a single mode, the line determined by the requirement $\operatorname{Im}(\omega)=0$ (Fig. 2) corresponds to a quasisteady-state mode which neither grows nor decays in time (that is, a true steady-state mode). For $\operatorname{Im}(\omega)<0$, the quasi-steady-state mode grows without limit, and for Im $(\omega)>0$, it decays to zero. When spontaneous emission [which was not included in (1)] is considered, the carrier densities of the laser are clamped just to the left of the Im $(\omega)=0$ line (in the absence of spontaneous emission, they are clamped onto it).

As we said, there are an infinite number of solutions Im $(\omega)=0$ to $(1)$, each corresponding to a different longitudinal mode; we plot a set of them in Fig. 3. Consider a point well to the left of any of the lines, e.g., point $A$ in Fig. 3. Then $\operatorname{Im}(\omega)>0$ for all the modes in the set; any excitation will decay away and no lasing state exists for that pair of carrier densities. On the other hand, a point on the curve's leftmost boundary (point $B$ ) is on the steady-state curve for one mode and in the $\operatorname{Im}(\omega)>0$ region for the rest; consequently, the one mode will lase (and since $\operatorname{Im}(\omega)=0$, neither grow nor decay) while all others decay away. An operating point at the intersection of two curves (point $C$ ) could conceivably have two lasing modes. However, a point to the right of any of the mode curves (point $D$ ) does not corresond to a physically

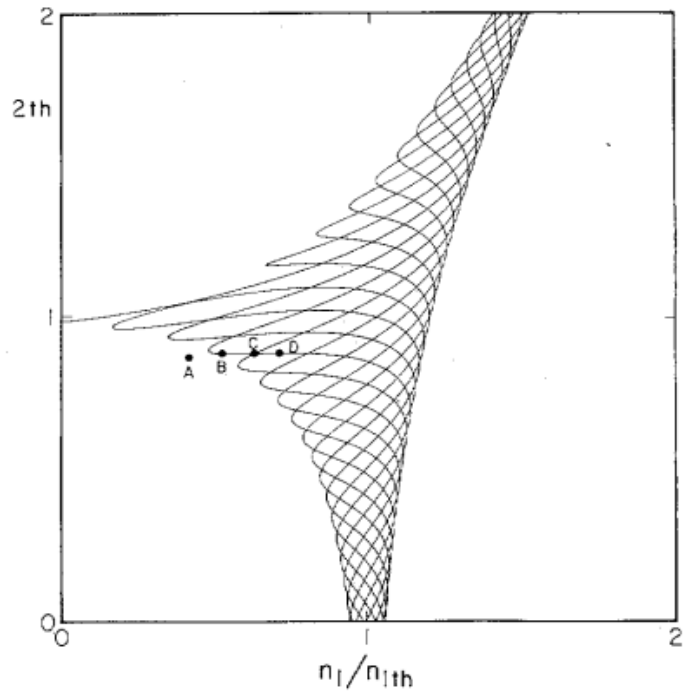

Fig. 3. Plot of ten modes in the carrier density plane.

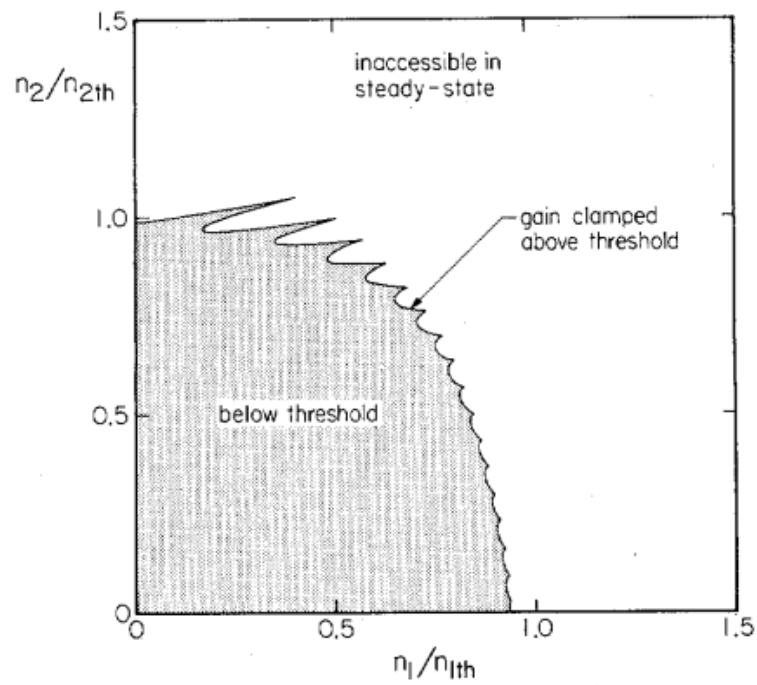

Fig. 4. Accessible regions of the carrier density plane. Above threshold, the carrier density is clamped onto the threshold carrier density line. Each cusp on the curve indicates a mode hop.

realizable steady state. It is in the $\operatorname{Im}(\omega)<0$ regime for one or more modes, and those modes will thus grow exponentially without limit. This region is, of course, accessible on a transient basis-but if one drives the device into the $\operatorname{Im}(\omega)<0$ region for a particular mode, the optical power in that mode will build up over time and saturate the gain back down to a point somewhere on the lowest curve. These arguments justify the division of the $\left(n_{1}, n_{2}\right)$ plane into regions below and above threshold as in Fig. 4. Between any two cusps, the curve corresponds to the $\operatorname{Im}(\omega)=0$ line of the lowest lying mode. The cusps correspond to mode hopping. Above lasing threshold, the carrier density is clamped onto the threshold line (although it is free to shift along the line).

In practice, one controls the currents supplied to the two sections, not the carrier densities. The question of multistability refers to the existence of multiple operating points $\left(n_{1}, n_{2}\right)$ for the same pump current densities $\left(j_{1}\right.$, 
$j_{2}$ ). We must also consider the carrier density equations

$$
\dot{n}_{i}=\frac{j_{i}}{q d}-\frac{n_{i}}{\tau_{s}}-g_{i}\left(n_{i}\right) \sum_{j} \Gamma_{i j} p_{j}
$$

where $q$ is the electronic charge, $d$ is the thickness of the active layer, $g_{i}\left(n_{i}\right)$ is the gain constant, and $\Gamma_{i j}$ are fill factors defined by

$$
\Gamma_{i j} \equiv\{\text { the proportion of optical power of the }
$$

$j$ th mode in the $i$ th cavity $\}.$

In steady state, we can write this equation as

$$
\frac{j_{i}}{q d}=\frac{n_{i}}{\tau_{s}}+g_{i}\left(n_{i}\right) \sum_{j} \Gamma_{i j} p_{j}
$$

Thus, for a given operating point $\left(n_{1}, n_{2}\right)$ and a given set of photon densities $p_{j}$, the currents are exactly determined. As we have seen, for all operating points well away from a mode boundary, only a single mode lases, so we can set the photon density for that mode equal to the total power density and set all of the other photon densities to 0 . For $p_{\text {tot }}=0$, the allowable currents are precisely equal to the threshold carrier densities (with a scaling factor of $q d / \tau_{s}$ ), and for $p_{\text {tot }}>0$, we can use (4) to replot the carrier density curves at any desired power level.

A modification occurs when we are at a cusp, since two lasing modes are possible. Let the two modes have power levels

$$
p_{1}=x p_{\text {tot }}, p_{2}=(1-x) p_{\text {tot }}
$$

so that $x$ is the relative fraction of optical power in mode 1 ; then the current relations can be written as

$$
\frac{j_{i}}{q d}=\frac{n_{i}}{\tau_{s}}+g_{i}\left(n_{i}\right)\left[x \Gamma_{i 1}+(1-x) \Gamma_{i 2}\right] p_{\mathrm{tot}} .
$$

It is clear from (6) that as $x$ is varied from 0 to 1 , the current (and mode) changes linearly from that of purely mode 1 to that of purely mode 2 . Hence, as we increase the power, the cusps which exist at threshold move out into the current plane and become straight line segments which join the single-mode curves. There are two qualitively different ways in which this situation manifests itself, both illustrated in Fig. 5 for a constant $p_{\text {tot }}$ (they usually do not occur together; both are shown on the same graph for illustrative purposes only). The line segment labeled "modes $1 \& 2$ "' is one of a family which fills the region of the current plane claimed [5] to possess no stable solution. The segment labeled "modes $2 \& 3$ " which completes the loop shows that multiple (in this case, three) steady-state solutions exist (the three solutions at three different power levels for the same $\left(j_{1}, j_{2}\right)$ are shown explicitly in Fig. 6). A steady-state analysis cannot tell us anything about the stability of such states, however. In the next section, we will transform the equations of motion near the mode hop to a simple system of nonlinear ordinary differential equations which incorporate all of the

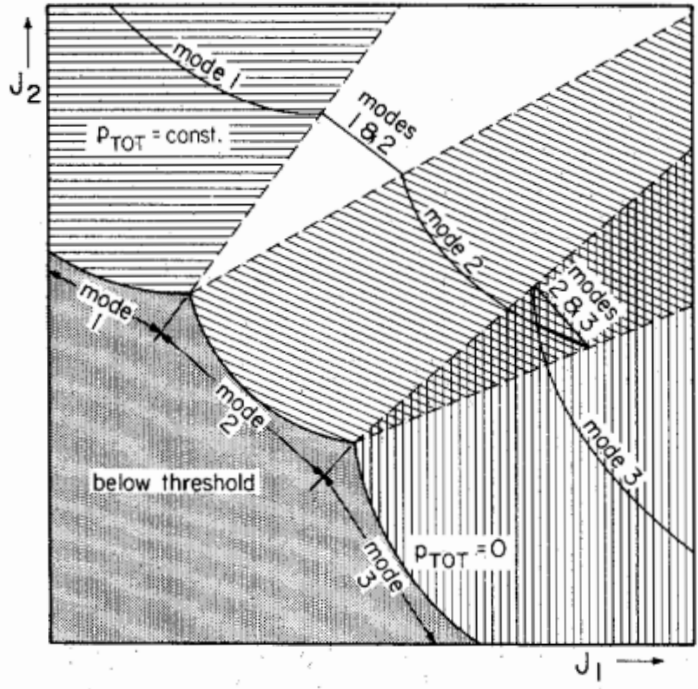

Fig. 5. Schematic of constant power curves in the current plane (arbitrary units). The $p_{\text {tot }}=0$ curve is identical to the threshold gain curve in the $\left(n_{1}, n_{2}\right)$ plane (within a scaling factor). The straight line segments joining the curves for fixed $p_{\text {to }} \geq 0$ are the mixed state (two simultaneously oscillating modes).

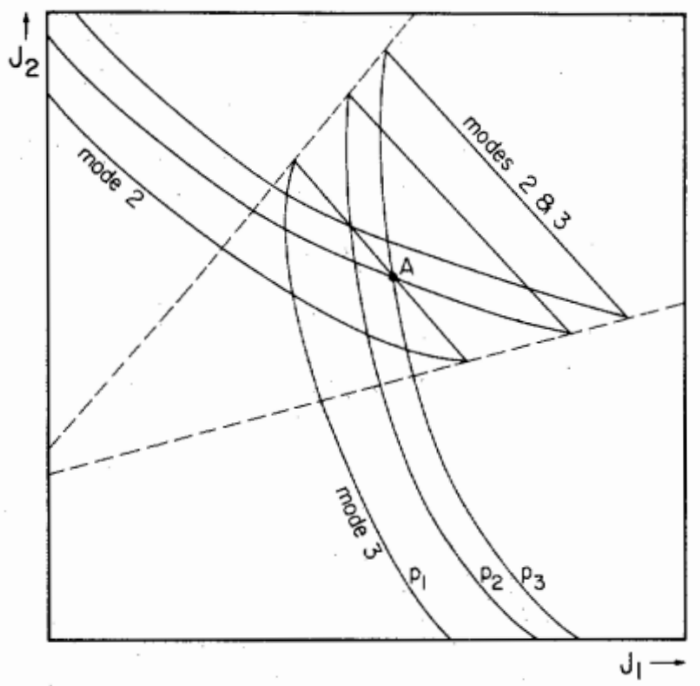

Fig. 6. Illustration of a single point in the current plane which possesses three steady-state solutions at different power levels (multimode at $p_{1}$, mode 2 at $p_{2}$, mode 3 at $p_{3}$ ).

relevant physics, yet allow simple analytic solutions and thus an unmistakeable interpretation of the physics of the device.

\section{Nondimensional Equations and Stability}

The problem as posed is strongly nonlinear; in particular, the variation of $\operatorname{Im}\left(\omega_{j}\right)$ with $n_{i}$ must be analyzed numerically. However, we can put (2) and (3) in nondimensional form valid near a mode boundary which carries all physical information without recourse to numerical techniques. The results we derive will be applicable to any two-element laser and not exclusively to a longitudinally coupled-cavity laser. 
We begin by putting (3) and (2) in matrix form:

$$
\begin{aligned}
& \dot{\tilde{N}}=\frac{1}{q d} \tilde{J}-\frac{1}{\tau_{s}} \tilde{N}-\tilde{\tilde{G}}(\tilde{N}) \tilde{\tilde{\Gamma}} \tilde{P} \\
& \dot{\tilde{P}}=\tilde{\tilde{W}} \tilde{P}+\tilde{\tilde{B}} \tilde{N} .
\end{aligned}
$$

The $i$ th element of $\tilde{N}$ is $\left\{n_{i}\right\}$, the elements of $\tilde{J}$ are $\left\{j_{i}\right\}$, the elements of $\tilde{\tilde{\Gamma}}$ are $\left\{\Gamma_{i j}\right\}$, the elements of $\tilde{\tilde{W}}$ are $\left\{\delta_{i j} \operatorname{Im}\left(\omega_{i}\right)\right\}$, the elements of $\tilde{\tilde{B}}$ are $\left\{\beta_{i} \delta_{i j} / \tau_{s}\right\}$, and the elements of $\tilde{P}$ are $\left\{p_{i}\right\}$. We now transform to the coordinate system $\tilde{M}$ illustrated in Fig. 7. If we linearize the mode lines about the mode crossing, then there exists a transformation matrix $\tilde{\tilde{R}}$ such that

$$
\tilde{M}=\tilde{\tilde{R}}\left(\tilde{N}-\tilde{N}^{(0)}\right)
$$

where the elements of $\tilde{N}^{(0)}$ are $\left\{n_{i}^{(0)}\right\}$, the coordinates of the mode crossing. (Note that $\tilde{\tilde{R}}$ is only unique to within a constant scaling matrix $\left(\begin{array}{cc}a & 0 \\ 0 & b\end{array}\right)$ with $a, b>0$; this ambiguity does not affect the analysis.) To lowest order, the system of matrix equations becomes

$$
\begin{aligned}
\dot{\tilde{M}} & =\frac{1}{q d} \tilde{\tilde{R}}\left(\tilde{J}-\frac{q d}{\tau_{s}} \tilde{N}^{(0)}\right)-\frac{1}{\tau_{s}} \tilde{M}-\tilde{\tilde{R}} \tilde{\tilde{G}}\left(\tilde{N}^{(0)}\right) \tilde{\tilde{\Gamma}} \tilde{P} \\
\dot{\tilde{P}} & =\tilde{\tilde{K}} \tilde{\tilde{M}} \tilde{P}+\tilde{\tilde{B}} \tilde{N}^{(0)}
\end{aligned}
$$

where the elements of $\tilde{K}$ are $\left\{-2\left(\partial \operatorname{Im}\left(\omega_{i}\right) / \partial m_{j}\right)\right\}$ and $\tilde{\tilde{M}}$ is the $2 \times 2$ matrix with the elements of $\tilde{M}$ on the diagonal. The virtue of choosing the axes $\left\{m_{i}\right\}$ is that (to the same order of approximation as the linearization) $\tilde{\tilde{K}}$ is diagonal. It is also important to note that with the axes so defined, det $\tilde{\tilde{R}}>0$ (if the axes were reversed, the sign would change). Now, we define dimensionless variables by taking

$$
\begin{aligned}
t & \equiv \tau_{s} \tau, \quad \tau \text { is the new time variable } \\
\tilde{E} & \equiv \tilde{\tilde{K}} \tilde{M} \\
\tilde{I} & \equiv \frac{\tau_{s}}{q d} \tilde{\tilde{K}} \tilde{\tilde{R}} \tilde{J}-\tilde{\tilde{K}} \tilde{\tilde{R}} \tilde{N}^{(0)} \\
\tilde{\tilde{H}} & \equiv \tilde{\tilde{K}} \tilde{\tilde{R}} \tilde{\tilde{G}}\left(\tilde{N}^{(0)}\right) \tilde{\tilde{\Gamma}} \\
\tilde{\tilde{D}} & \equiv \tilde{\tilde{B}} \tilde{N}^{(0)}
\end{aligned}
$$

which reduces (10) and (11) to the simple system

$$
\begin{aligned}
& \tilde{E}=\tilde{I}-\tilde{E}-\tilde{\tilde{H}} \tilde{P} \\
& \tilde{P}=\tilde{E} \tilde{P}+\tilde{D} .
\end{aligned}
$$

In component form,

$$
\begin{aligned}
& \dot{e}_{i}=i_{i}-e_{i}-\sum_{j} h_{i j} p_{j} \\
& \dot{p}_{j}=e_{j} p_{j}+d_{j} .
\end{aligned}
$$

Equations (14) and (15) are several levels removed from the original system, so it is helpful to review the terms in each equation and identify them with a physical mechanism. $\left\{e_{i}\right\}$ are carrier densities, $\left\{i_{i}\right\}$ are pump currents,

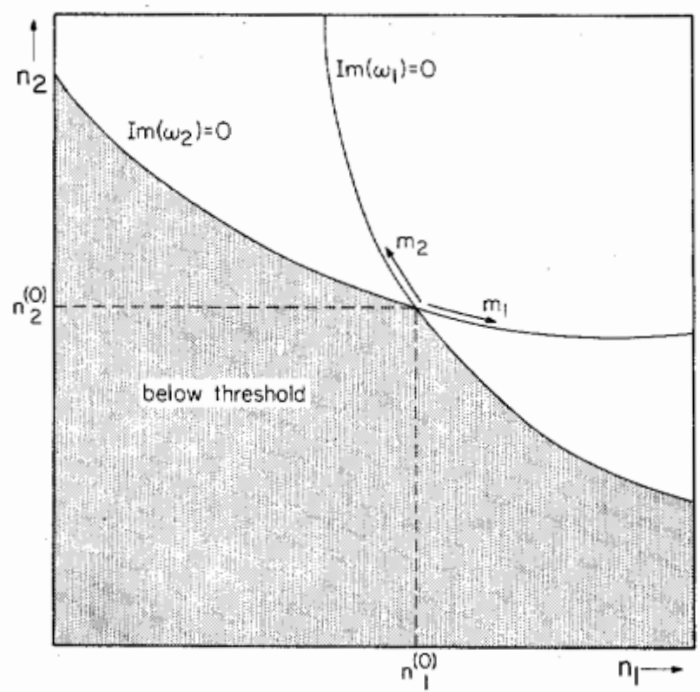

Fig. 7. Schematic of a single mode crossing in the carrier density plane (arbitrary units). The transformed variables $\left\{m_{i}\right\}$ are measured along the two intersecting mode lines.

$\left\{p_{i}\right\}$ are the modal power densities, $\left\{h_{i j}\right\}$ are the fill factors (the proportion of mode $j$ in carrier pool $i$ ), and $\left\{d_{j}\right\}$ are the spontaneous emission rates. Thus, the three terms on the right side of (14) correspond to pump current, spontaneous emission, and stimulated emission, respectively; the two terms on the right of (15) are stimulated emission and spontaneous emission, respectively. Examination of $(12 \mathrm{~d})$ reveals that $\operatorname{det} H$ is of the same sign as det $\Gamma$ (because the determinants of all of the intervening matrices is positive). This is indicative of the fact that in transforming from the $\left\{n_{i}\right\}$ representation to the $\left\{e_{i}\right\}$ representation, we "stretched" the axes, but did not flip them over. Also, we note that the spontaneous emission rate is typically quite small $\left(\beta_{i} \approx 10^{-4}\right)$ so that $\left\{d_{i}\right\}$ are also of the same small order.

The steady-state solutions of (14) and (15) are plotted in the current plane for a fixed $p_{\text {tot }}$ in Fig. 8 (subject to the physical constraint $p_{i} \geq 0$ ). The solutions in the absence of spontaneous emission $\left(d_{i}=0\right)$ are apparent by inspection (one virtue of the dimensionless system of equations). They are

$$
\left\{p_{1}=0, e_{2}=0, e_{1}\right. \text { free }
$$

or

$$
\left\{p_{2}=0, e_{1}=0, e_{2}\right. \text { free }
$$

or

$$
\left\{e_{1}=e_{2}=0, p_{2}=p_{\text {tot }}-p_{1}, p_{1}\right. \text { free. }
$$

If we include spontaneous emission and define $x$ as the free parameter (restricted to $x \in[0,1]$ ), the solutions are

$$
\left\{\begin{array}{l}
p_{1}=x p_{\mathrm{tot}}, p_{2}=(1-x) p_{\mathrm{tot}}, \\
e_{1}=\frac{-d_{1}}{x p_{\mathrm{tot}}}, e_{2}=\frac{-d_{2}}{(1-x) p_{\mathrm{tot}}} .
\end{array}\right.
$$

Equation (17) is more exact than (16); however, the shape of the curves is not as obvious from the equations. 


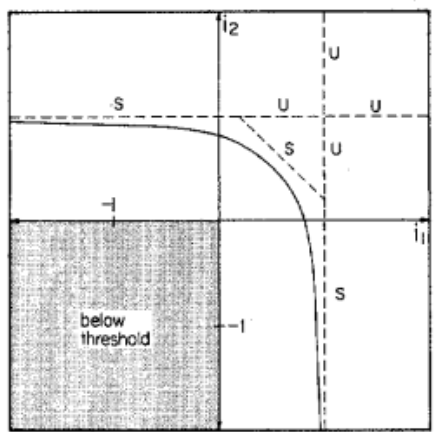

(a)

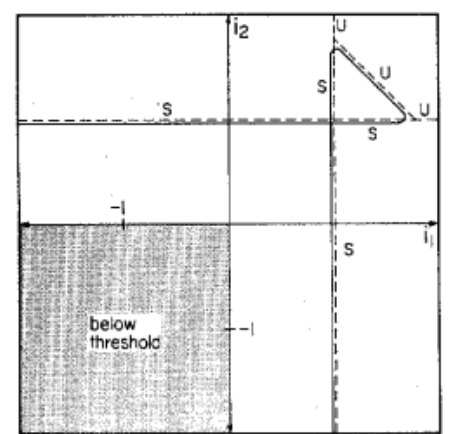

(b)
Fig. 8. Steady-state modes in the transformed current plane for fixed total output power $p_{\text {tot }}=1$. Dotted lines are solutions in the absence of spontaneous emission $\left(d_{i} \equiv 0\right)$, solid lines are solutions including spontaneous emission $\left(d_{1}=d_{2}=0.05\right)$. (a) $h_{11}=h_{22}=1, h_{12}=h_{21}=0.2$, so det $H \geq 0$. (b) $h_{11}=h_{22}=1, h_{12}=h_{21}=1.8$, so det $H \leq 0$.

With or without spontaneous emission, we still must prove that these steady-state solutions are stable. We do this by performing a second linearization of (14) and (15) and determining the boundedness of the response of the homogeneous system to a perturbation.

$$
e_{i} \rightarrow e_{i}+\epsilon_{i}, p_{i} \rightarrow p_{i}+\rho_{i}
$$

giving

$$
\begin{aligned}
& \dot{\epsilon}_{i}=-\epsilon_{i}-\sum_{j} h_{i j} \rho_{j} \\
& \dot{\rho}_{j}=\epsilon_{j} p_{j}+e_{j} \rho_{j} .
\end{aligned}
$$

These can be put into matrix form as

$$
\left(\begin{array}{l}
\epsilon_{1} \\
\epsilon_{2} \\
\rho_{1} \\
\rho_{2}
\end{array}\right)=\left(\begin{array}{cccc}
-1 & 0 & -h_{11} & -h_{21} \\
0 & -1 & -h_{12} & -h_{22} \\
p_{1} & 0 & e_{1} & 0 \\
0 & p_{2} & 0 & e_{2}
\end{array}\right)\left(\begin{array}{c}
\epsilon_{1} \\
\epsilon_{2} \\
\rho_{1} \\
\rho_{2}
\end{array}\right) \text {. }
$$

The solution to (20) is bounded only if all of the eigenvalues of the square matrix have nonpositive real parts. The secular equation for the eigenvalue $\lambda$ is

$$
\begin{gathered}
(\lambda+1)^{2}\left(\lambda-e_{1}\right)\left(\lambda-e_{2}\right)+(\lambda+1)\left(\lambda-e_{2}\right) p_{1} h_{11} \\
+(\lambda+1)\left(\lambda-e_{1}\right) p_{2} h_{22}+p_{12} \operatorname{det} H=0
\end{gathered}
$$

We shall first solve for the case with no spontaneous emission.

Case $I-e_{2}=p_{1}=O$ (Only Mode 2 Lasing): Equation (21) reduces to

$$
(\lambda+1)\left(\lambda-e_{1}\right)\left[\lambda(\lambda+1)+p_{2} h_{22}\right]=0 .
$$

The roots are $\lambda=-1, \lambda=e_{1}, \lambda=-\left(\frac{1}{2}\right) \pm\left[\frac{1}{4}-\right.$ $\left.p_{2} h_{22}\right]^{1 / 2}$, so the solution is stable if $e_{1} \leq 0$.

Case II- $e_{1}=p_{2}=0$ (Only Mode 1 Lasing): By switching subscripts we see that $e_{2} \leq 0$ is necessary for stability.

Case III- $e_{1}=e_{2}=O$ (Multimode State):

$$
\begin{gathered}
(\lambda+1)^{2} \lambda^{2}+\left(p_{1} h_{11} p_{2} h_{22}\right) \lambda(\lambda+1) \\
+p_{1} p_{2} \operatorname{det} H=0 .
\end{gathered}
$$

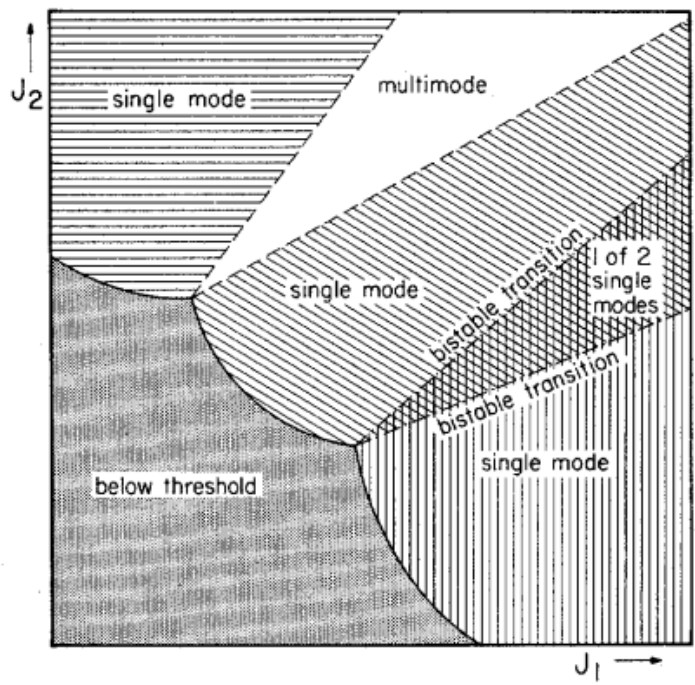

Fig. 9. Division of the current plane into regions of subthreshold lasing, multimode operation, single-mode operation, and bistable operation. Lines of instability on which bistable transitions take place are indicated.

Define $x \equiv \lambda(\lambda+1)$. Then

$$
\lambda=-\frac{1}{2} \pm\left[\frac{1}{4}+x\right]^{1 / 2}
$$

so $x$ must have a negative real part for stability. The equation for $x$ is

$$
x^{2}+\left(p_{1} h_{11}+p_{2} h_{22}\right) x+p_{1} p_{2} \operatorname{det} H=0 .
$$

From inspection, we can see that $x$ has a negative real part only if det $H \geq 0$. In Fig. 8, we have plotted representative cases in the $\left(i_{1}, i_{2}\right)$ plane for the two possible signs of det $H$. Case I yields the vertical dashed lines, Case II yields horizontals, and Case III yields the diagonal line; det $H \geq 0$ in Fig. 8(a) and det $H \leq 0$ in Fig. 8(b). Thus, the multimode state (Case III) is stable in Fig. 8(a), while it is unstable in Fig. 8(b). All of the steady-state solutions in Fig. 8 are labeled ( $S=$ stable, $U=$ unstable) according to these rules. While one can work out the roots for the case including spontaneous emission numerically, there is no need; as the spontaneous emission goes to zero, the solution lines and the roots of (21) must collapse onto those of no spontaneous emission. Therefore, the labelings are valid for the curves which include spontaneous emission.

One important point is that the portions of the singlemode curves in Fig. 8(b) which overlap each other (and consequently yield multiple solutions) are stable; bistable transitions can only occur at their endpoints (where they become unstable). Furthermore, we have proven the stability of the mixed state of Fig. 8(a). It remains to relate this nondimensional problem to the physical problem with which we started. This is accomplished by noting that the point $\left(e_{1}, e_{2}\right)=(0,0)$ is the crossing of the two mode lines in Fig. 5. Since (as we pointed out earlier) det $H$ and det $\Gamma$ have the same sign, the stability criterion for the mixed state is that det $\Gamma \geq 0$. The different states and their stability are summarized in Fig. 9. The current plane will, in general, divide into regions of single-mode operation; the boundaries will either consist of bimodal re- 
gions (two simultaneous lasing modes) where det $\Gamma \geq 0$ or bistable regions (two possible single-mode states) where det $\Gamma \leq 0$. This is contrary to the assertions of [5]. One final point of interest: the stable mixed state [Fig. 8(a)] is a multimode state that persists in the absence of spontaneous emission. This is to be contrasted with the more usual multimode behavior where the intensity of side modes is proportional to the spontaneous emission rate. (It should be pointed out that with insufficient selectivity between modes, any laser-including coupled cavity-will run multimode due to the spontaneous emission.)

There are several features of this behavior which would be of interest from a systems viewpoint. Every point in the bimodal region corresponds to the same set of carrier densities $\left\{e_{1}=e_{2}=0\right\}$. Therefore, the carrier density is fixed, and so the gain and resonant refractive index are effectively clamped (which is not the case in a single-mode region). Consequently, if one modulates the laser entirely within the multimode region, there is no direct frequency modulation of either of the two modes. With the addition of a notch filter to remove one of the two modes from the optical output, the device becomes a chirpless modulator. Also the response time of a laser diode is dependent upon the total optical power present. By modulating the diode in the bimodal region, the optical power is merely switched from one mode to another (as in a push-pull amplifier); consequently, even though the amplitude of the desired mode may approach zero, the total power present remains constant and the response time of the device remains short.

On the other hand, if the cavity were tuned such that $\operatorname{det} \Gamma \leq 0$ at the mode hop, the device possesses electrical bistability and the inherent noise immunity associated with bistability. Examination of (4) shows that an external beam of light impinging upon one of the cavities shifts the entire set of tuning curves up and down (or side to side) in the current plane. Hence, all-optical bistability is equally feasible.

\section{Conclusions}

In summary, we have analyzed the steady-state operation of a two-element coupled-cavity semiconductor laser near a mode boundary. We showed how the equations of motion for a specific system of a longitudinally coupledcavity laser can be reduced to a nondimensional set of nonlinear differential equations which describe the behav- ior of a general two-element coupled-cavity laser near a mode boundary. We showed that a multimode state which was previously unexplained exists whenever the determinant of the matrix of fill-factor coefficients is nonzero. The multimode state varies continuously between the two involved single modes regardless of the level of spontaneous emission.

If the determinant is greater than zero, the multimode state is stable. Otherwise, it is unstable, but there are two stable single-mode states. Bistable transitions occur on the boundaries of the unstable multimode state. Large bistabilities are desirable from the point of optical storage and hysteretic devices; consequently, the relation between the bistable region and the size of the determinant of the fillfactor matrix is a useful design criterion for such devices.

\section{REFERENCES}

[1] W. T. Tsang and N. A. Olsson, "High speed direct single-frequency modulation with large tuning rate and frequency excursion in cleavedcoupled-cavity semiconductor lasers," Appl. Phys. Lett., vol. 42, pp. $650-652,1983$.

[2] K. J. Ebeling, L. A. Coldren, B. I. Miller, and J. A. Rentschler, "'Single-mode operation of coupled-cavity GaInAsP/InP semiconductor lasers," Appl. Phys. Lett., vol. 42, pp. 6-8, 1983.

[3] I. H. White, J. E. Carroll, and R. G. Plumb, "Closely coupled twinstripe lasers," Proc. IEE, vol. 129, pp. 291-293, 1982.

[4] Ch. Harder, K. Y. Lau, and A. Yariv, "Bistability and negative resistance in semiconductor lasers," Appl. Phys. Lett., vol. 40, pp. 124-126, 1982.

[5] C. H. Henry and R. F. Kazarinov, "Stabilization of single frequency operation of coupled cavity lasers," IEEE J. Quantum Electron., vol. QE-20, pp. 733-744, 1984.

[6] D. Marcuse and T.-P. Lee, "Rate equation model of a coupled-cavity laser," IEEE J. Quantum Electron., vol. QE-20, pp. 166-176, 1984.

[7] K. J. Ebeling and L. A. Coldren, "Analysis of multielement semiconductor lasers,"' J. Appl. Phys., vol. 54, pp. 2962-2969, 1983.

[8] W. Streifer, D. Yevick, T. L. Paoli, and R. D. Burnham, "An analysis of cleaved coupled-cavity lasers," IEEE J. Quantum Electron., vol. QE-20, pp. 754-764, 1984.

[9] J. Salzman, R. J. Lang, and A. Yariv, "Laterally coupled cavity semiconductor laser," Appl. Phys. Lett., vol. 47, pp. 195-197, 1985.

[10] R. J. Lang and A. Yariv, "Analysis of the dynamic response of multielement semiconductor lasers," IEEE J. Quantum Electron., vol. QE-21, pp. 1683-1688, 1985 .

Robert J. Lang (S'83), for a photograph and biography, see p. 448 of the March 1986 issue of this JOURNAL.

Amnon Yariv (S'56-M'59-F'70), for a photograph and biography, see $\mathrm{p}$. 448 of the March 1986 issue of this Journal. 